\title{
The dual FXR/TGR5 agonist INT-767 counteracts nonalcoholic steatohepatitis in a rabbit model of high fat diet-induced metabolic syndrome
}

\section{Vignozzi, I Cellai, S Filippi, P Comeglio, E Sarchielli, A Morelli, E Maneschi, GB Vannelli, L Adorini, M Maggi}

\author{
Department of Experimental and Clinical Biomedical Sciences, University of Florence, Florence, Italy
}

\section{Introduction and objectives}

Farnesoid X receptor (FXR) and Takeda G protein-coupled receptor 5 (TGR5) are interesting pharmacological targets for the treatment of liver and metabolic diseases. FXR-deficient mice on a high-fat diet (HFD) exhibit massive hepatic steatosis, necro-inflammation and fibrogenesis. Moreover, pharmacological activation of TGR5 in mice promotes protective mechanisms in biliary epithelial cells, inhibits hepatic and systemic inflammation.

The aim of this study is to investigate the effect of FXR/TGR5 dual agonists on nonalcoholic steatohepatitis (NASH) in a rabbit model of high fat diet (HFD)-induced MetS.

\section{Methods}

We employed a recently established animal model of high fat diet (HFD)-induced MetS, characterized by insulin resistance, hypertension, atherogenic dyslipidemia, visceral adipose tissue accumulation and NASH (Filippi et al., 2009, Maneschi et al., 2013). Subgroups of MetS rabbits were treated with increasing doses of the dual FXR/TGR5 agonist INT-767 $(3,10,30 \mathrm{mg} / \mathrm{Kg}$, orally, daily, 5 days a week for 12 weeks). We studied the effects of HFD and in vivo INT-767 treatments on liver function. Liver was studied by immunohistochemistry and RT-PCR.

\section{Results}

- Treatment with increasing doses of the dual FXR/TGR5 agonist INT$767(3,10,30 \mathrm{mg} / \mathrm{Kg} /$ day, 5 days a week for 12 weeks) in a rabbit model of HFD-induced MetS, characterized also by NASH, dosedependently reduced several MetS-associated alterations, including hepatomegaly, insulin resistance, increase of ALT, glucose and cholesterol levels, while significantly increasing HDL levels. ALT was positively associated with all MetS parameters; however introducing all MetS factors in a multivariate analysis, only total cholesterol levels resulted positively associated with ALT level (Adj.r: 0.493, $p=0.014$ ). High macrophage M1pro-inflammatory/M2 anti-inflammatory ratio was observed in MetS-induced NASH, which was independently associated with serum ALT levels (Adj.r: 0.322, $p=0.032$ ). HFD-induced increase in M1/M2 ratio was reduced by INT-767 treatment and M2 macrophage markers (IL-10,TGF $\beta$ ) were increased.

Genes related to neutrophil apoptosis/apoptotic-neutrophil clearance (lactoferrin, eNOS, RAGE) and to extracellular matrix degradation (MMP2, TIMP2) were also increased by INT-767 treatment. INT-767 also reduced liver expression of IL-6, which preferentially skews the Th cell response towards a Th17-phenotype, while increasing Foxp3 expression, a Treg cell marker (Fig. 1). Thus these data indicate that INT-767 can promote the neutrophil- and macrophage-driven resolution phase of inflammation and fibrosis regression. In addition, INT-767 increased genes related to hepatic fatty acid metabolism (PPAR $\boldsymbol{\alpha}, \mathbf{A R}, \mathbf{C D} 36$ ) and lipid droplet formation (SNAP23, VAMP4, syntaxin5, perilipin) therefore suggesting that INT-767 counteracts excess fatty acid mediated lipotoxicity in the liver. Genes related to insulin signaling (IRS1, SREBP1, G6Pase, and PEPCK) were also increased by INT-767 (Fig. 1).

- Finally, immunohistochemical studies demonstrated that INT-767 treatment significantly reduced both HFD-induced liver inflammation (Fig. 2) and fibrosis. (Fig. 3)

\section{2)}
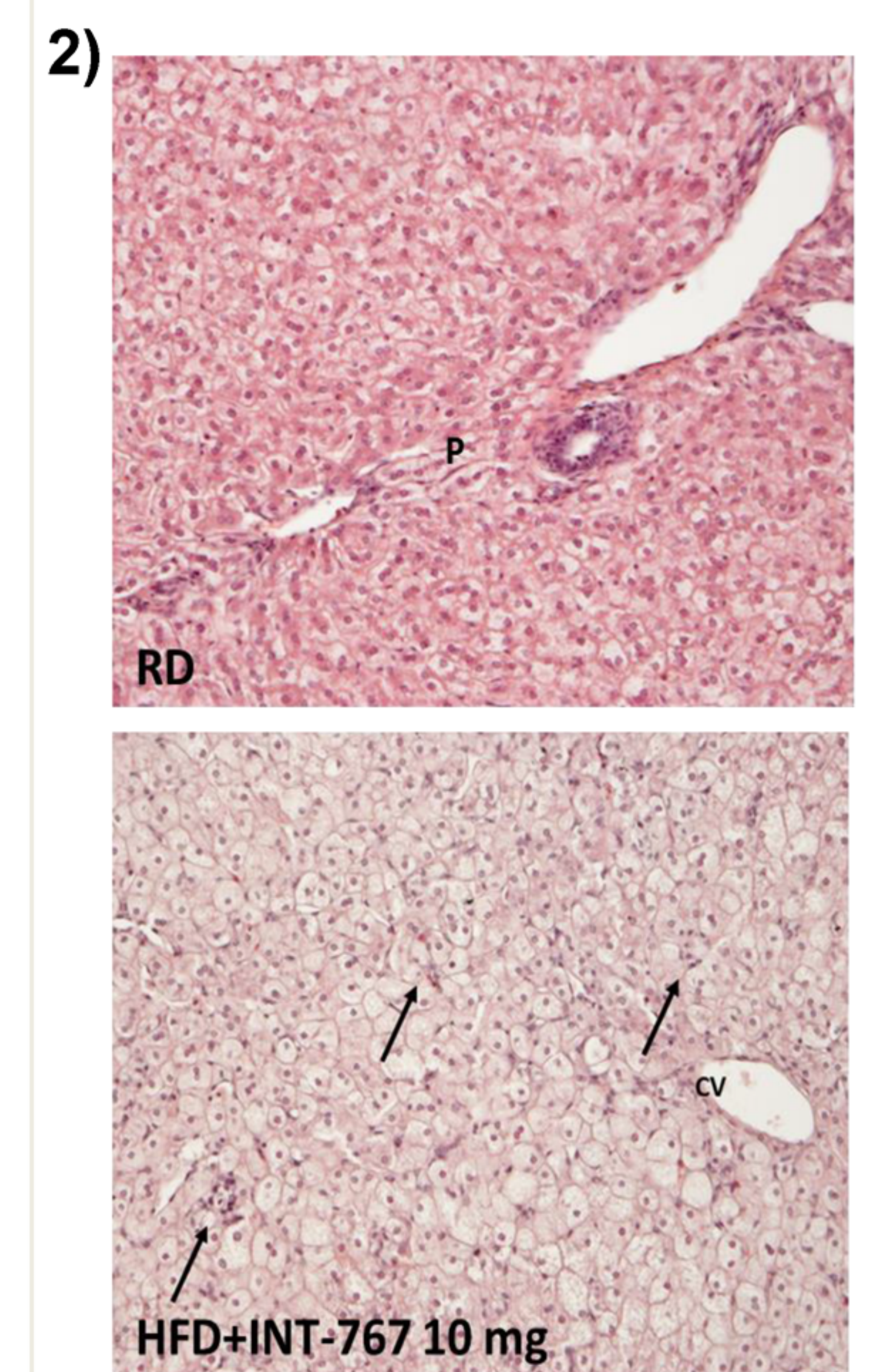

Magnification 10x
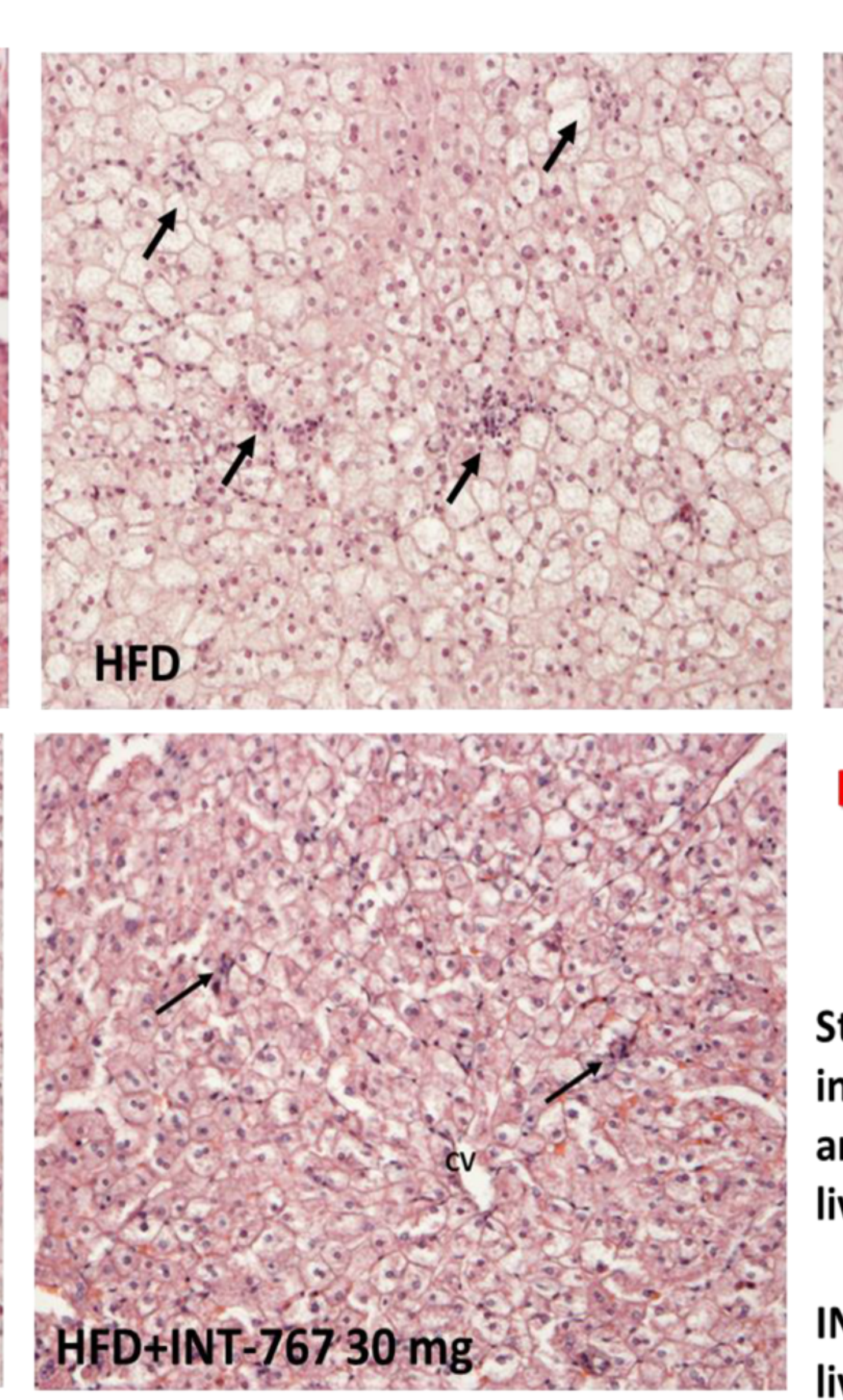

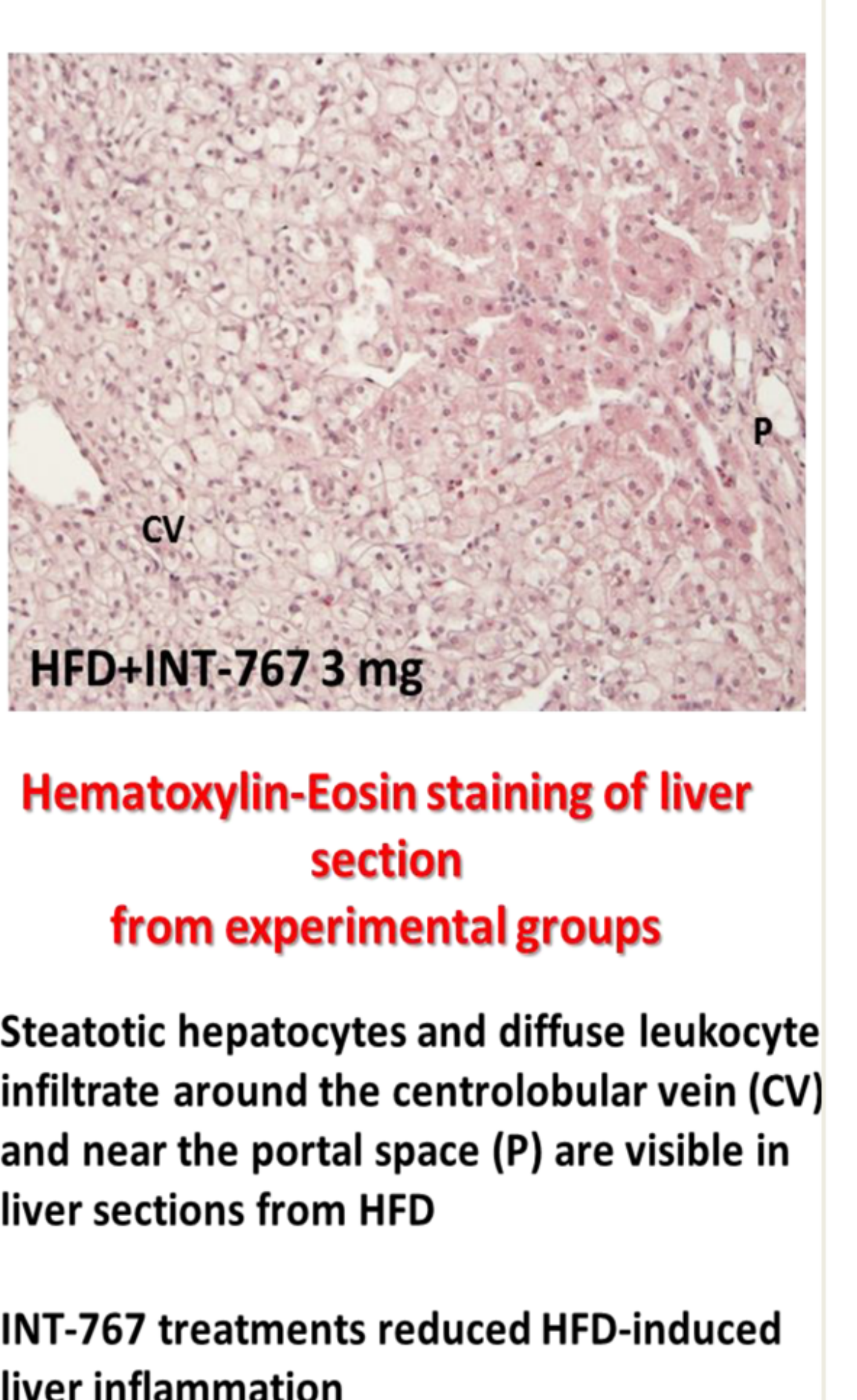

liver inflammation
1)

\begin{tabular}{|c|c|c|c|c|c|c|}
\hline & & RD & HFD & $\begin{array}{l}\text { HFD+INT-767 } \\
3 \mathrm{mg}\end{array}$ & $\begin{array}{l}\text { HFD+INT-767 } \\
10 \mathrm{mg}\end{array}$ & $\begin{array}{l}\text { HFD+INT-767 } \\
30 \mathrm{mg}\end{array}$ \\
\hline & M1/M2 ratio & $1.1 \pm 0.2$ & $219.9 \pm 24.5^{* * *}$ & $62.6 \pm 9.4^{\circ 0}$ & $34 \pm 16^{\circ 0}$ & $27.1 \pm 12^{\circ 00}$ \\
\hline \multirow{3}{*}{$\begin{array}{l}\text { M2 macrophage } \\
\text { markers }\end{array}$} & {$[\mathrm{IL} 10$} & $100 \pm 8.69$ & $1218.28 \pm 143.19^{* * *}$ & $* 2779.2 \pm 560.50^{\circ 0}$ & $2125.1 \pm 831.1^{\circ}$ & $2486.4 \pm 774.3^{\circ}$ \\
\hline & TGF $\beta$ & $100 \pm 6.48$ & $389.02 \pm 37.03^{* * *}$ & $1416.45 \pm 228.28^{\circ 00}$ & $693.3 \pm 204.5$ & $1064.3 \pm 405.7^{\circ 0}$ \\
\hline & lactoferrin & $100 \pm 10.58$ & $83.09 \pm 23.29 * *$ & $95.52 \pm 54.95$ & $107.5 \pm 57.7$ & $30.2 \pm 21.7$ \\
\hline \multirow{2}{*}{$\begin{array}{l}\text { Neutrophil apoptosis } \\
\text { markers }\end{array}$} & eNOS & $100 \pm 5.95$ & $116.38 \pm 11.70$ & $279.52 \pm 38^{\circ 0}$ & $255.56 \pm 21.34^{\circ 0}$ & $311 \pm 40.8^{\circ 00}$ \\
\hline & RAGE & $100 \pm 19.21$ & $434.93 \pm 63.55^{* * *}$ & $813.96 \pm 123.17^{\circ}$ & $379.7 \pm 36.7$ & $514.4 \pm 105.3$ \\
\hline \multirow{2}{*}{$\begin{array}{l}\text { Extracellular matrix } \\
\text { degradation markers }\end{array}$} & MMP2 & $100 \pm 6.48$ & $4095.79^{* * *}$ & $10264 \pm 2497.28^{\circ 0}$ & $2348.4 \pm 872.2$ & $2779.4 \pm 815.9$ \\
\hline & TIMP2 & $100 \pm 7.09$ & $954.77 \pm 118.80^{* * *}$ & $2021.2 \pm 140.55^{\circ 0}$ & $1128.2 \pm 352.3$ & $1336.8 \pm 307$ \\
\hline Th17 cell marker & {$[$ IL-6 } & $100 \pm 13.33$ & $215.36 \pm 33.77 * *$ & $82.89 \pm 49.37$ & $49.55 \pm 8.04^{\circ}$ & $105.5 \pm 51.5$ \\
\hline \multirow[t]{2}{*}{ Treg cell marker } & Foxp3 & $100 \pm 13.41$ & $1438.8 \pm 225.62$ & $3188.2 \pm 1414.39^{\circ}$ & $766.4 \pm 544$ & $332.1 \pm 103.7$ \\
\hline & [PPARa & $100 \pm 4.11$ & $71.28 \pm 6.11^{* * *}$ & $173.33 \pm 11.89^{\circ 00}$ & $214.9 \pm 26.3^{\circ 00}$ & $193.4 \pm 44.1^{\circ 0}$ \\
\hline \multirow{3}{*}{$\begin{array}{l}\text { Hepatic fatty acid } \\
\text { Metabolism markers }\end{array}$} & $A R$ & $100 \pm 4.67$ & $118.78 \pm 11.85$ & $211.39 \pm 13.88^{\circ}$ & $179.2 \pm 31.2$ & $252.2 \pm 34.3^{\circ 0}$ \\
\hline & CD36 & $100 \pm 5.03$ & $98 \pm 10.50$ & $313.21 \pm 32.91^{000}$ & $179.5 \pm 33.4^{\circ}$ & $156.3 \pm 43$ \\
\hline & SNAP23 & $100 \pm 6.80$ & $125.93 \pm 15.11$ & $404.97 \pm 23.46^{\circ 0}$ & $294.6 \pm 43.9^{\circ 0}$ & $302 \pm 28.9^{000}$ \\
\hline \multirow{3}{*}{$\begin{array}{c}\text { Lipid droplet } \\
\text { Formation markers }\end{array}$} & VAMP4 & $100 \pm 7.26$ & $95.99 \pm 9.45$ & $194.84 \pm 9.01^{\circ 0}$ & $151.5 \pm 28.7$ & $187.7 \pm 13.05^{\circ 0}$ \\
\hline & Syntaxin 5 & $100 \pm 4.63$ & $104.39 \pm 9.68$ & $294.53 \pm 37.62^{\circ \circ}$ & $209.4 \pm 24.3^{\circ 0}$ & $221.6 \pm 22.7^{000}$ \\
\hline & Perilipin & $100 \pm 16.38$ & $255.29 \pm 48.62^{* * *}$ & $568.76 \pm 63.83^{\circ}$ & $761.4 \pm 250.8$ & $1557.3 \pm 625.9$ \\
\hline \multirow{4}{*}{$\begin{array}{l}\text { Insulin signaling } \\
\text { markers }\end{array}$} & IRS1 & $100 \pm 6.21$ & $150.79 \pm 19.06^{* *}$ & $268.60 \pm 33.07^{\circ}$ & $231.6 \pm 38.5$ & $298.9 \pm 7^{000}$ \\
\hline & SREBP1 & $100 \pm 9.30$ & $202.77 \pm 33.01^{* * *}$ & $557.49 \pm 80.17^{\circ 0}$ & $310.5 \pm 108.7$ & $306.1 \pm 104.6$ \\
\hline & G6Pase & $100 \pm 7.84$ & $37.81 \pm 4.85 * * *$ & $259.07 \pm 58.26^{000}$ & $247.51 \pm 33.7^{000}$ & $184.4 \pm 61.1^{000}$ \\
\hline & PEPCK & $100 \pm 4.62$ & $53.71 \pm 5.18^{* * *}$ & $157.71 \pm 3.68^{000}$ & $158.24 \pm 12.1^{100}$ & $160 \pm 35.5^{\circ 00}$ \\
\hline
\end{tabular}

In conclusion, INT-767 treatment counteracts NASH in a rabbit model of HFD-induced MetS by promoting insulin sensitivity, resolution of inflammation and fibrosis regression.

3)
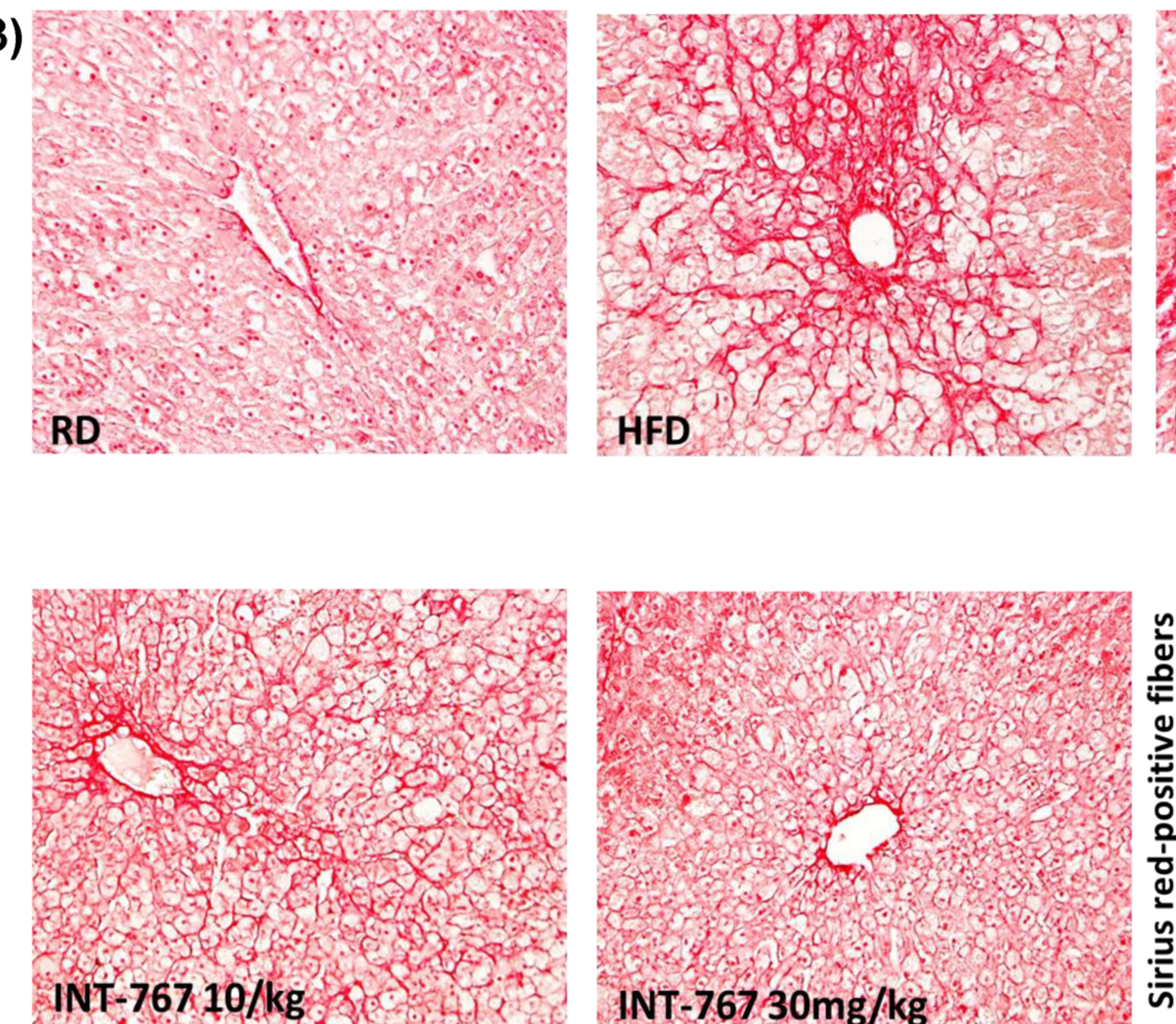

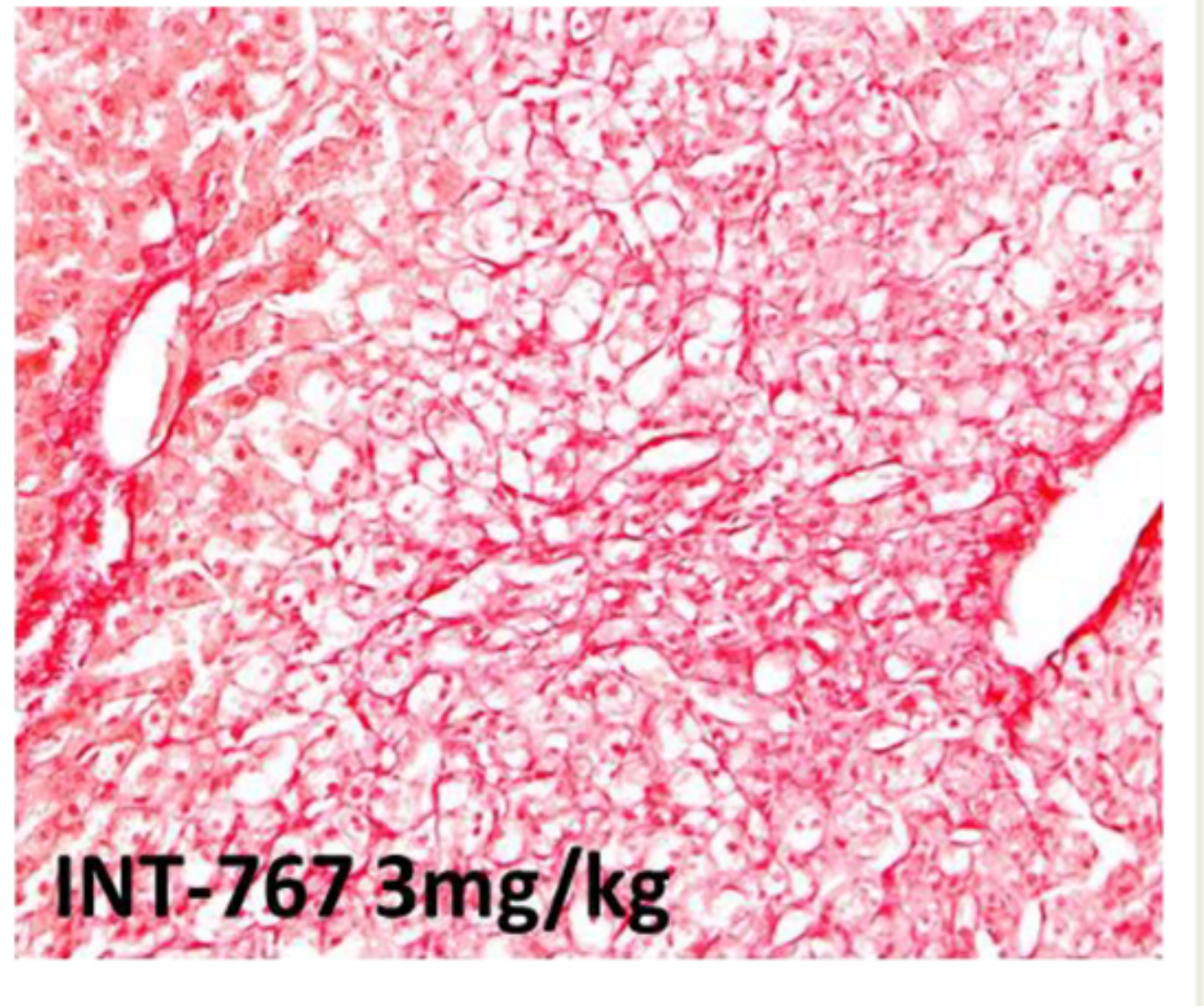

${ }^{*} \mathrm{p}<0.05,{ }^{* *} \mathrm{p}<0.001$ vs RD; ${ }^{\circ} p<0.05,{ }^{\circ} 0.001$ vs. HFD

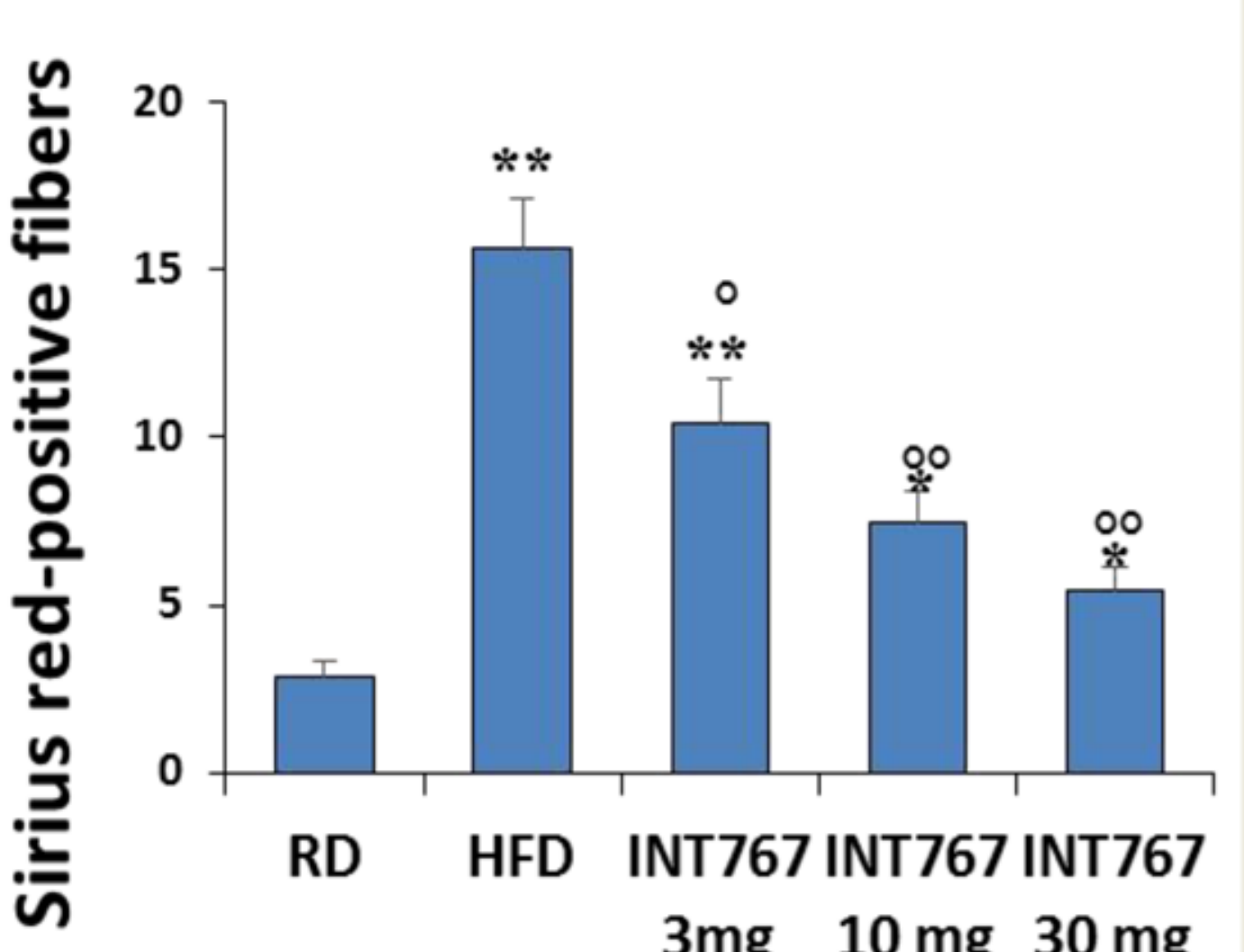

-1) Filippi S, Vignozzi L, Morelli A, Chavalmane AK, Sarchielli E, Fibbi B, Saad F, Sandner P, Ruggiano P, Vannelli GB, Mannucci E, Maggi M (2009) Testosterone partially ameliorates metabolic profile and erectile responsiveness to PDE5inhibitors in an animal model of male metabolic syndrome. J Sex Med6(12):3274-88. doi: 10.1111/j.1743-6109.2009.01467.x

-2) Maneschi E, Vignozzi L, Morelli A, Mello T, Filippi S, Cellai I, Comeglio P, Sarchielli E, Calcagno A, Mazzanti B, Vettor R, Vannelli GB, Adorini L, Maggi M (2013) FXR activation normalizes insulin sensitivity in visceral preadipocytes of a rabbit model of MetS. J Endocrinol218(2):215-31. doi: 10.1530/JOE-13-0109. 